\title{
Mechanisms responsible for surfactant changes in sepsis-induced lung injury
}

\author{
W. Huang, L.A. McCaig, R.A.W. Veldhuizen, L-J. Yao and J.F. Lewis
}

\begin{abstract}
Pulmonary surfactant is altered in sepsis, and these changes contribute to the predisposition of septic lungs to subsequent insults, ultimately leading to acute lung injury. Specifically, the total amount of surfactant is lower in sepsis, mainly due to decreased small aggregate (SA) surfactant pools. The amount of large aggregate (LA) surfactant is not altered.

To evaluate the mechanisms responsible for these alterations, trace doses of tritium-labelled dipalmitoylphosphatidylcholine $\left({ }^{3} \mathrm{H}-\mathrm{DPPC}\right)$-labelled LA were instilled intratracheally into adult rats $20 \mathrm{hrs}$ after caecal ligation and perforation (CLP) or sham surgery. Animals were sacrificed at 0,1 and $4 \mathrm{~h}$ after instillation and recovery of ${ }^{3} \mathrm{H}$-DPPC in alveolar macrophages (AM), LA and SA was measured. In separate in vitro experiments, AM isolated from CLP/sham rats were incubated with LA or SA isolated from normal animals to evaluate the uptake of these aggregates into the AM.

Results showed increased surfactant radioactivity associated with AM of CLP animals compared with sham animals both in vivo and in vitro. In addition, more ${ }^{3} \mathrm{H}$-DPPC label remained in LA forms in the CLP animals in vivo compared with sham.

These findings indicate that differences in surfactant aggregate uptake and large aggregate conversion occur in septic lungs, resulting in changes in surfactant pools.
\end{abstract}

KEYWORDS: Lung injury, sepsis, surfactant

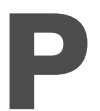
ulmonary surfactant is a mixture of phospholipids and proteins that reduces surface tension at the air-liquid interface of the alveoli and thus prevents alveolar collapse during end-exhalation [1]. The surfactant system is altered both quantitatively and functionally in patients with acute lung injury (ALI) [2-6]. Furthermore, these observed impairments of pulmonary surfactant contribute to lung dysfunction. Unfortunately, the results of clinical trials evaluating exogenous surfactant administration in patients with ALI have been disappointing, in part due to the complexity and severity of the injury at the time of treatment [7-9]. Although earlier interventions would seem appropriate, a better understanding of surfactant alterations at this stage of the disease is required to justify this approach. Recent studies have shown that changes in the alveolar surfactant subfractions occur in animals and humans with less severe lung injury, while spontaneously breathing [10-12].

Within the alveoli, surfactant is present in a functional large aggregate (LA). During respiration, the LA are converted into nonfunctional smaller vesicles called small aggregates (SA) [13]. While the amounts of these aggregates remain stable within the normal lung, in severely injured lungs, the relative percentage of LA is decreased
$[3,14]$. Interestingly, recent findings have shown an increase in the percentage of large aggregate forms at earlier stages of injury. For example, the current authors have shown that both rats and mice with a mild lung injury induced by sepsis had an increase in the percentage of LA, which was due to a decrease in SA pools within the airspace $[10,15]$. The mechanisms responsible for these specific changes are unknown, but did not involve surfactant protein A (SP-A) nor inducible nitric oxide synthase $[15,16]$.

The current authors hypothesised that the alterearlier stages of sepsis, were due to an increased uptake of surfactant by alveolar macrophages and/or a decreased conversion of large into small aggregates. To test this hypothesis, the current authors determined the fate of intratracheally injected tritium labelled dipalmitoylphosphatidylcholine $\left({ }^{3} \mathrm{H}\right.$-DPPC) LA in rats after caecal ligation and perforation (CLP). In addition, the uptake of radiolabelled aggregates by alveolar macrophages (AM) isolated from CLP and sham animals was evaluated in vitro.

\section{MATERIALS AND METHODS}

\section{Preparation of radiolabelled aggregates}

LA were isolated via differential centrifugation of lung lavage obtained from normal adult rats. The ations of endogenous surfactant, occurring at
AFFILIATIONS

Dept of Physiology and

Pharmacology and Medicine, Lawson

Health Research Institute, the

University of Western Ontario,

London, Ontario, Canada.

\section{CORRESPONDENCE}

J.F. Lewis

Dept of Physiology and

Pharmacology and Medicine

Lawson Health Research Institute

University of Western Ontario

London

ON N6A 4V2

Canada

Fax: 15196466064

E-mail: jflewis@uwo.ca

Received:

July 222005

Accepted after revision:

August 152005

SUPPORT STATEMENT

These studies were funded by a grant from the Canadian Institutes of Health Research.

Online ISSN 1399-3003 
LA were then labelled with ${ }^{3} \mathrm{H}-\mathrm{DPPC}$ and the association of the radiolabel with the LA was verified with the use of sucrose density centrifugation, as previously described [17]. Radiolabelled SA were obtained via surface area cycling of aliquots of labelled LA [18]. Briefly, aliquots of labelled LA were isolated and cycled at $40 \mathrm{rev} \cdot \mathrm{min}^{-1}$, at $37^{\circ} \mathrm{C}$, for $180 \mathrm{~min}$. SAs were obtained in the supernatant of a $15 \mathrm{~min}, 40,000 \times \mathrm{g}$ centrifugation, which were quantified by measuring phospholipids-phosphorus via the method of DucK-CHONG [19] after chloroform extraction [20], and by measurement of radioactivity by scintillation counting.

\section{Animal procedure}

The animal procedures were performed according to the guidelines of the Canadian Council on Animal Care and were approved by the University Animal Care Committee. Sepsis was induced via the CLP procedure in adult rats as described previously [10]. Briefly, male Sprague-Dawley rats (350-450 g) were anaesthetised and the right external jugular vein and right carotid artery were cannulated with PE50 tubes. Both catheters were then routed subcutaneously to the back of the neck and attached to a three-fluid-channel (22-gauge) swivel system that allowed the rat to have an unlimited movement within the cage. A laparotomy was performed and animals randomised to the CLP procedure had the distal one-third section of the caecum ligated, punctured twice with a 16-gauge needle and compressed to extrude bowel contents into peritoneum. The caecum was then returned to the abdominal cavity and the incision was closed with 2.0 sutures. Sham animals underwent identical procedures after induction of anaesthesia, including catheter placement and laparotomy, but the CLP procedure was not performed.

After surgery, the rats were placed in plastic cages to recover. All animals received a continuous infusion of sterile saline at $7.5 \mathrm{~mL} \cdot \mathrm{kg}^{-1} \cdot \mathrm{h}^{-1}$ containing $2 \mu \mathrm{g} \cdot \mathrm{mL}^{-1}$ of fentanyl for analgesia via the venous catheter. The arterial catheter was infused with sterile heparinised saline $\left(1 \mathrm{U} \cdot \mathrm{mL}^{-1}\right)$ at $1 \mathrm{~mL} \cdot \mathrm{h}^{-1}$ to maintain patency. Arterial blood gases were measured on an Acid-Base Laboratory 500 blood gas analyser (Radiometer, Copenhagen, Denmark). Arterial lactate levels were measured via a YSI 2300 STAT plus glucose/lactate analyser (Yellow Springs Instruments, Yellow Springs, OH, USA). Mean arterial pressure (MAP) and heart rate (HR) were recorded via a pressure transducer attached to the arterial line. Respiratory rate (RR) was also recorded. All of these parameters were measured at 4 and $20 \mathrm{~h}$ post surgery.

\section{In vivo metabolism of large aggregates}

At 20-h post sham or CLP surgery, the rats were reanaesthetised intravenously with ketamine $\left(10-20 \mathrm{mg} \cdot \mathrm{kg}^{-1}\right)$ and xylazine $\left(0.5-1 \mathrm{mg} \cdot \mathrm{kg}^{-1}\right)$. After sedation was induced, the neck incision was reopened and the trachea was exposed. The animal was placed on a board at a $45^{\circ}$ angle, and a trace dose of ${ }^{3} \mathrm{H}$-DPPC-labelled LA $\left(0.5 \mu \mathrm{Ci} \cdot \mathrm{kg}^{-1}\right.$ body weight in $0.5 \mathrm{~mL}$ of saline, $0.5 \mu \mathrm{Ci}=0.125 \mathrm{mg}$ phospholipids) was intratracheally instilled through a 22.5-gauge needle. After closing the incision with 2.0 sutures, the rats were then placed back into their cages and allowed to recover until sacrifice. Groups of animals were sacrificed at 0,1 , and $4 \mathrm{~h}$ post LA radiolabelled instillation via an overdose of sodium pentobarbital $\left(110 \mathrm{mg} \cdot \mathrm{kg}^{-1}\right)$ followed by transection of the abdominal aorta.

After sacrifice, the lungs were lavaged five times with saline, as previously described [10]. The total lavage fluid was then centrifuged at $150 \times g$ for $10 \mathrm{~min}$ to obtain the cellular pellet. The $150 \times g$ cell pellet was suspended in $1 \mathrm{~mL}$ of saline with aliquots of this suspension taken for cell counting via a haemocytometer, and for cell differential counting on a cytocentrifuge slide stained by the HemaColor ${ }_{\circledast}$ (EM Science, Gibbstown, NJ, USA). After washing twice with saline, the remaining cell pellet was subsequently resuspended in $200 \mu \mathrm{L}$ lysis buffer $(150 \mathrm{mM} \mathrm{NaCl}, 50 \mathrm{mM} \mathrm{NaPO}, 2 \mathrm{mM}$ EDTA, and $0.5 \%$ Nonidet P-40) for scintillation counting for radioactivity recovery. In the present study, cell differential counts demonstrated that the cell population isolated from the lung lavage of both CLP and sham rats was $>98 \%$ macrophages. Therefore, the radioactivity measurements in the cell pellets were regarded as that attributed totally by the AM. The $150 \times g$ supernatant of each animal's lavage was then centrifuged at $40,000 \times g$ for $15 \mathrm{~min}$, yielding a supernatant containing the SA subfraction of alveolar surfactant. The $40,000 \times g$ pellet was suspended in saline and defined as the LA fraction.

Immediately after the lavage procedure, the lung tissue was removed and homogenised in $15 \mathrm{~mL}$ saline, with aliquots extracted by the method of BLIGH and DYER [20]. The radioactivity of the lung homogenate extract together with aliquots of the alveolar cell suspension (150×g pellet), the $150 \times g$ supernatant (total surfactant), both LA and SA fractions as well as an aliquot of the administered radiolabelled input sample were all processed for scintillation counting. The total recovery of administrated radioactivity was expressed as the sum recovered in alveolar cells, total surfactant, and lung homogenate relative to the administered input sample.

To measure surfactant pool sizes in the CLP and sham animals at the 20-h time point after surgery, aliquots from the total surfactant, LA and SA fractions were extracted and phospholipid levels were evaluated using a modification of the DuckChong phosphorous assay $[19,20]$. Total protein in the $150 \times g$ supernatant (total surfactant) sample was determined by the microbicin-choninic acid method (Pierce, Rockford, IL, USA) with bovine serum albumin used as the standard.

\section{In vitro uptake of aggregates by alveolar macrophages}

Separate groups of CLP/sham animals were prepared as described above and killed $20 \mathrm{~h}$ after surgery with their lungs subsequently lavaged. The cell pellet was isolated by centrifuging the lavage at $150 \times g$ for $10 \mathrm{~min}$, and washed twice with ice-cold saline. The cells were then counted on a haemocytometer and viability was determined via trypan blue exclusion. Under the conditions employed, neither sepsis nor the sham surgical procedure affected the viability of lavage cells. The isolated cells ( $>98 \%$ macrophages) were resuspended in Dulbeco's Modified Eagle's Medium (Gibco Invitrogen, Burlington, On, Canada) with $10 \%$ foetal bovine serum at a concentration of $1 \times 10^{6}$ cells $\cdot \mathrm{mL}^{-1}$. Aliquots of normal, fresh ${ }^{3} \mathrm{H}$ DPPC labelled LA or SA as described in the preparation of radiolabelled aggregates above, were added to this cell suspension at a concentration of $10 \mu \mathrm{g}$ phospholipid $\cdot \mathrm{mL}^{-1}$. The cells were then incubated at $37^{\circ} \mathrm{C}$ for $60 \mathrm{~min}$ with gentle 
agitation. Lipid uptake of the AM was stopped by washing and centrifuging the cells three times with ice-cold saline. After the third centrifugation, the cell pellet was suspended in lysis buffer for measurement of radioactivity via scintillation counting.

\section{Statistics}

Data are presented as means \pm SE. Values among groups were analysed using a two-way ANOVA followed by the unpaired Newman-Keuls t-test. A probability level of $p<0.05$ was considered statistically significant.

\section{RESULTS \\ Physiological parameters of caecal ligation and perforation/sham rats}

There were no differences in body weights between the CLP and sham rats ( $422 \pm 7 \mathrm{~g}$ versus $423 \pm 10 \mathrm{~g}, \mathrm{n}=15$ per group). At the 4-h time point, post surgery, there were no significant differences in arterial blood gases (arterial oxygen tension: $\mathrm{Pa}, \mathrm{O}_{2}$; carbon dioxide arterial tension: $\left.\mathrm{Pa}_{1} \mathrm{CO}_{2} ; \mathrm{pH}\right)$, alveolararterial oxygen tension difference $\left(\mathrm{PAa}_{2} \mathrm{O}_{2}\right)$, arterial lactate levels, MAP, respiratory rate (RR) and HR between the two groups (table 1). These data indicate that all animals adequately recovered from the surgical procedures up to 4-h post surgery. Data shown at $20 \mathrm{~h}$ represent the values recorded immediately prior to the animals being re-anaesthetised for intratracheal instillation of the labelled LA. Consistent with previous studies in both rats and mice undergoing similar CLP procedures, significantly lower $\mathrm{Pa}_{2} \mathrm{O}_{2}, \mathrm{MAP}$, and significantly higher $P A a, O_{2}, H R, R R$, and arterial lactate levels were observed in the CLP rats, compared with the sham group (table 1) [10, $15,16]$. Consistent with these changes, representing a relatively mild lung injury, were previous studies showing that there were no changes in static lung compliance in the CLP group at $20 \mathrm{~h}$ as measured by pressure-volume curves $[10,15,16]$.

\begin{tabular}{|c|c|c|c|c|}
\hline \multirow[t]{3}{*}{ TABLE 1} & $\begin{array}{l}\text { siological } \\
\text { surgery }\end{array}$ & aramete & for rats a & 4 and $20 \mathrm{~h}$ \\
\hline & \multicolumn{2}{|c|}{ Sham } & \multicolumn{2}{|c|}{ CLP } \\
\hline & $4 h$ & $20 \mathrm{~h}$ & $4 \mathrm{~h}$ & $20 \mathrm{~h}$ \\
\hline $\mathrm{Pa}, \mathrm{O}_{2} \mathrm{mmHg}$ & $95.5 \pm 1.6$ & $94.5 \pm 1.3$ & $93.2 \pm 2.2$ & $82.7 \pm 3.4^{\star \star, \#}$ \\
\hline $\mathrm{Pa}, \mathrm{CO}_{2} \mathrm{mmHg}$ & $33.2 \pm 0.7$ & $36.9 \pm 0.8^{\#}$ & $34.1 \pm 1.0$ & $39.9 \pm 1.4^{\#}$ \\
\hline $\mathrm{O}_{2}$ saturate $\%$ & $95.4 \pm 0.6$ & $95.8 \pm 0.6$ & $94.3 \pm 0.7$ & $92.5 \pm 0.7^{* *}$ \\
\hline$P A a, \mathrm{O}_{2}$ & $12.5 \pm 1.4$ & $8.8 \pm 1.2$ & $14.5 \pm 2.0$ & $17.9 \pm 3.3^{*}$ \\
\hline MAP mmHg & $118 \pm 2$ & $124 \pm 2$ & $114 \pm 3$ & $116 \pm 3^{*}$ \\
\hline HR beats $\min ^{-1}$ & $343 \pm 8$ & $338 \pm 7$ & $335 \pm 7$ & $372 \pm 14^{*, \#}$ \\
\hline RR breaths $\min ^{-1}$ & $91 \pm 2$ & $94 \pm 2$ & $92 \pm 1$ & $106 \pm 4^{\star \star * \#}$ \\
\hline Lactate $\mathrm{mmol} \cdot \mathrm{L}^{-1}$ & $0.61 \pm 0.03$ & $0.62 \pm 0.03$ & $0.69 \pm 0.02$ & $1.0 \pm 0.07^{* *, \#}$ \\
\hline
\end{tabular}

Data presented as mean $\pm \mathrm{SD}, \mathrm{n}=15$. CLP: caecal ligation and perforation; $\mathrm{Pa}_{1} \mathrm{O}_{2}$ : arterial oxygen tension; $\mathrm{Pa}_{\mathrm{a}} \mathrm{CO}_{2}$ : carbon dioxide arterial tension; $\mathrm{PAa}_{2} \mathrm{O}_{2}$ : alveolar-arterial oxygen tension difference; MAP: mean arterial pressure; HR: heart rate; RR: respiratory rate. ${ }^{*}: p<0.05,{ }^{*} p<0.01$ versus sham; ${ }^{*}: p<0.01$ versus 4-h time point.

\section{Alveolar surfactant pools, cells and total protein}

The number of AM recovered from the CLP rats was not significantly different from that recovered from sham animals

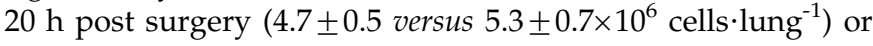
at various time points after intratracheal instillation of the trace dose of radiolabelled LA (data not shown). Figure 1 shows the total surfactant and aggregate pool sizes for CLP and sham rats $20 \mathrm{~h}$ post surgery. Both total surfactant and SA pool sizes were significantly lower in CLP versus sham groups $(\mathrm{p}<0.01)$, but LA pools were not different. Similar results were observed for samples obtained after the trace dose of labelled LAs were instilled (data not shown). Total protein levels in lung lavage were similar in CLP $\left(17.6 \pm 2.9 \mathrm{mg} \cdot \mathrm{kg}^{-1}\right)$ and sham $\left(17.7 \pm 2.3 \mathrm{mg} \cdot \mathrm{kg}^{-1}\right)$ groups at all time points, indicating minimal leak of plasma proteins into the airspace at this early and relatively mild phase of sepsis-induced lung injury.

\section{Tritium dipalmitoylphosphatidylcholine recovery in vivo}

Immediately after the intratracheal instillation of radiolabelled LA at the 20-h time point after either the CLP or sham procedure (time $0 \mathrm{~h}$, fig. 2), the total recovery of the radiolabel in the lung (lavage+lung tissue+alveolar macrophages) relative to the input sample was $70.5 \pm 3.2$ and $72.1 \pm 2.7 \%$ in the CLP rats and sham rats, respectively. This suggests that some of the instilled radiolabel was lost during the instillation and processing procedures. The recovery of the radiolabel in the lavage and lung tissue, as shown in figure $2 \mathrm{a}$, is expressed as a percentage of the radioactivity measured at the 0 -h time point. Although recovery in lavage decreased and recovery in tissue increased over time after instillation, there were no statistically significant differences noted between the CLP and sham groups in the radiolabel recovery. Figure $2 b$ shows that the radioactivity recovered in AM normalised to cell number. The radioactivity associated with the alveolar macrophages isolated from the CLP rats was significantly higher than the activity associated with the macrophages isolated from the sham rats. This was statistically significantly high at the 1-h $\left(\mathrm{p}<0.05\right.$, ) and 4 -h time points $(\mathrm{p}<0.01)$ after ${ }^{3} \mathrm{H}$-DPPC LA was instilled.

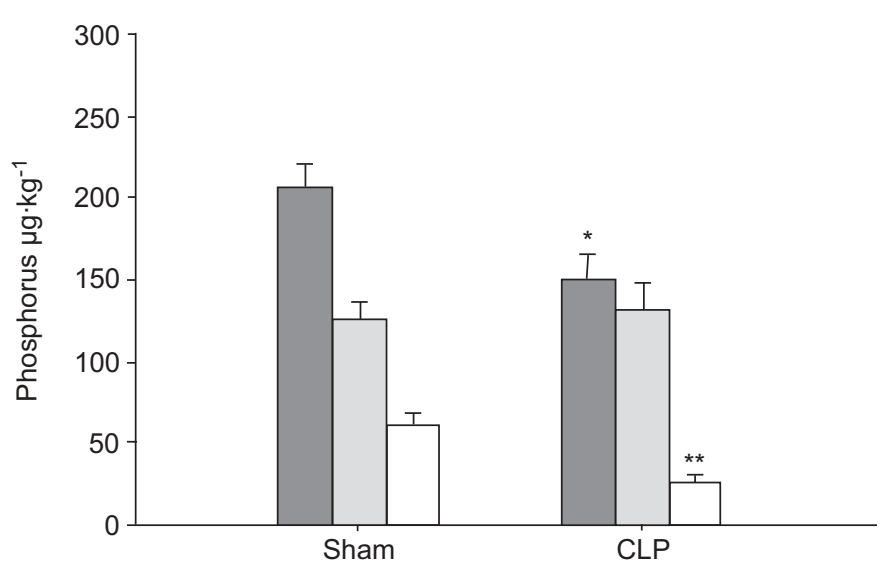

FIGURE 1. Analysis of phosphorus-phospholipid in alveolar lavage of caecal ligation and perforation (CLP)/sham group rats 20-h post surgery. Animals were sacrificed, lungs were lavaged and surfactant was isolated and analysed. $\square$ : total surfactant; $\square$ : large aggregate; $\square$ : small aggregate. Results are shown as means $\pm S E, n=8 ; *: p<0.05 ; * *: p<0.01$ versus the sham group. 

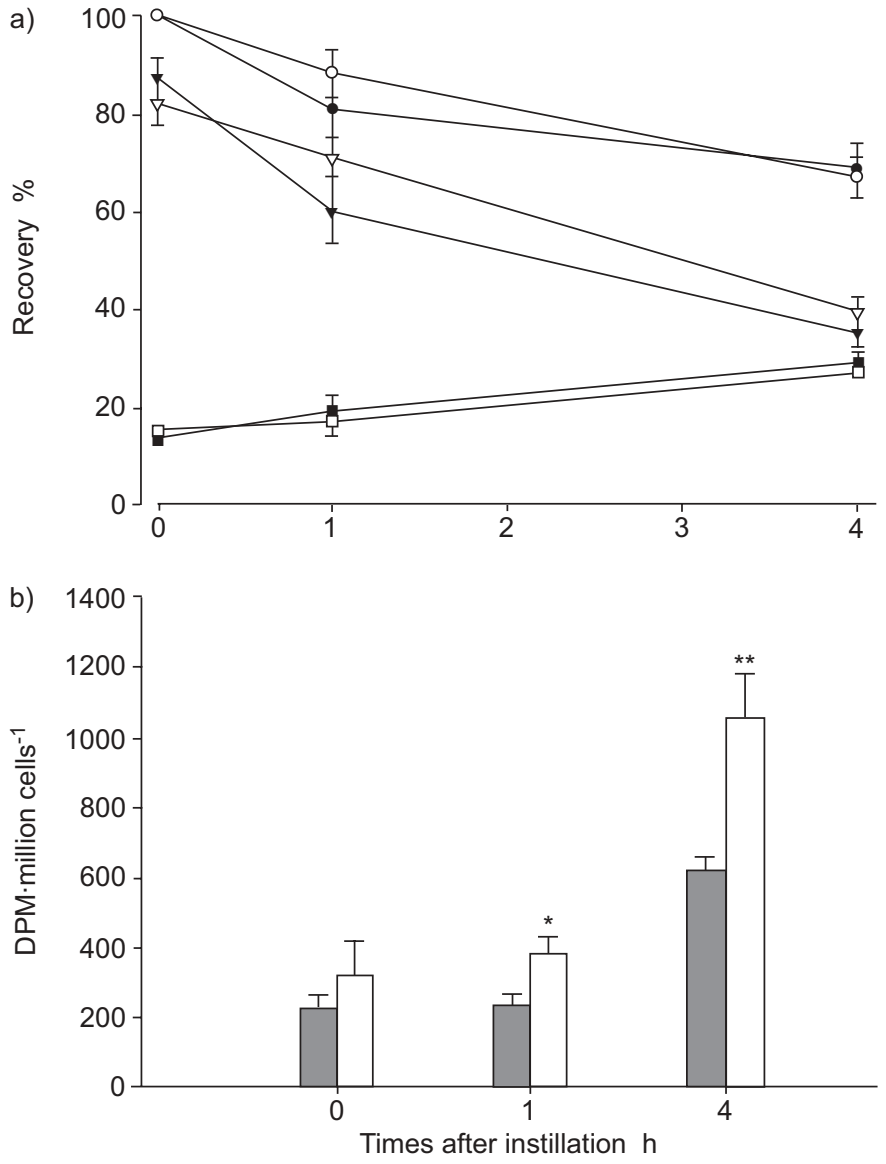

FIGURE 2. Recovered radioactivity in the lung of caecal ligation and perforation (CLP)/sham rats after the intratracheal instillation of tritium-labelled dipalmitoylphosphatidylcholine ( ${ }^{3} \mathrm{H}$-DPPC) large aggregates (LA). ${ }^{3} \mathrm{H}$-DPPClabelled LA was intratracheally instilled into the lung of sham/CLP rats at $20 \mathrm{~h}$ post surgery. Animals were then killed and lungs were lavaged at 0-, 1-, and 4-h time points after instillation ( $\mathrm{n}=5$ per time point per group). a) The per cent recovery of radioactivity in alveolar total surfactant (TS), lung tissue relative to the recovery of total lung at $0 \mathrm{~h}$ was shown. $\bullet$ : sham rat total lung; $\mathrm{O}$ : CLP rat total lung; $\mathbf{\nabla}$ : sham rat lavage TS; $\nabla$ : CLP rat lavage TS; $\mathbf{\square}$ : sham rat lung tissue; $\square$ CLP rat lung tissue. b) The recovered radioactivity in alveolar macrophages was represented as disintegrations per minute (DPM) per million cells. Results are presented as means $\pm S E, n=5$. $\square$ : sham rats; $\square$ : CLP rats. *: $p<0.05$; **: $p<0.01$ versus sham group at corresponding time point.

Figure 3 shows the per cent recovery of ${ }^{3} \mathrm{H}$-labelled LA in the lavage obtained from both CLP and sham animals at 0,1 and $4 \mathrm{~h}$ after instillation. There was progressively less of the ${ }^{3} \mathrm{H}$ DPPC recovered as LA over time $(0-4 \mathrm{~h})$ in both groups, although at the 4-h time point, a significantly higher per cent recovery of LA was observed in the CLP group compared with the corresponding sham group $(\mathrm{p}<0.05)$.

\section{Tritium dipalmitoylphosphatidylcholine uptake in vitro}

Figure 4 shows the results of the in vitro experiments involving the incubation of normal radiolabelled aggregates with AM isolated from either sham or CLP animals. The amount of radiolabelled LA associated with AM after 60 min of incubation with either sham or CLP AM was significantly greater

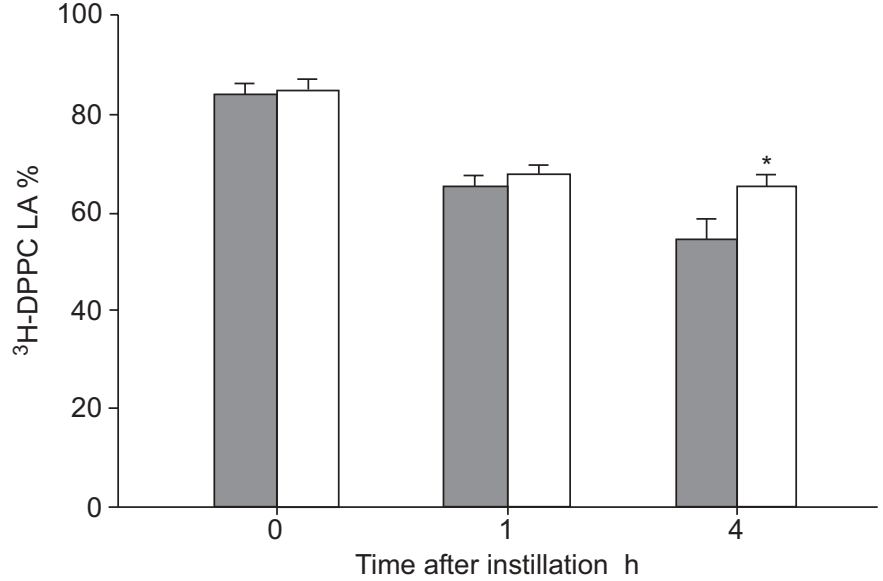

FIGURE 3. The per cent recovery of radiolabel in the large fraction of lung lavage after instillation. Animal groups and treatments were the same as described in figure 2. Alveolar large aggregate $(L A)$ and small aggregate fractions were separated by centrifugation and radioactivity was counted. Results are means $\pm \mathrm{SE}$, $\mathrm{n}=5 .{ }^{3} \mathrm{H}$-DPPC: titrium-labelled dipalmitoylphospatidylcholine. : : large aggregate; $\square$ : small aggregate*: $p<0.05$ versus sham group at corresponding time point.

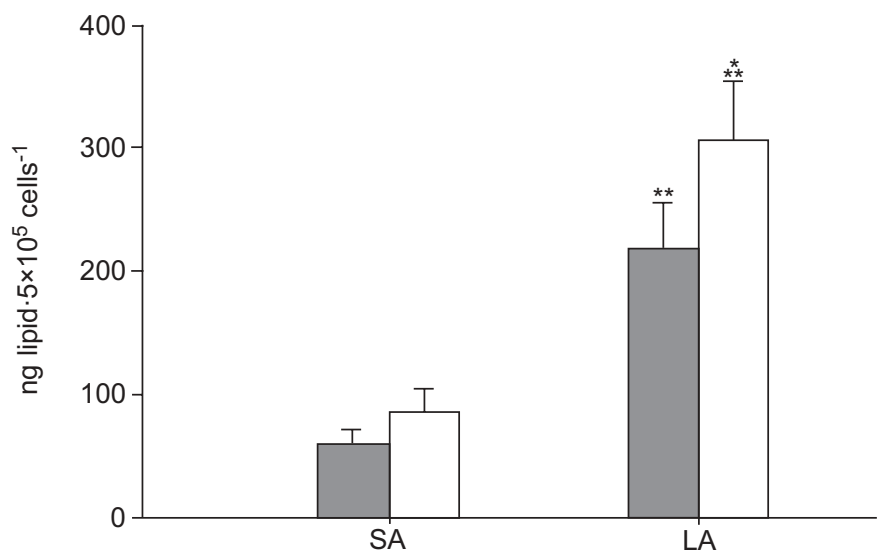

FIGURE 4. In vitro uptake of small aggregates (SA) and large aggregates (LA) fractions by alveolar macrophages from caecal ligation and perforation (CLP) or sham rats after $20 \mathrm{~h}$. The isolated cells were incubated with ${ }^{3} \mathrm{H}$-DPPC-labelled LA or ${ }^{3} \mathrm{H}$-DPPC-labelled SA at a concentration of $10 \mu \mathrm{g}$ phospholipids $\cdot \mathrm{mL}^{-1}$ for $60 \mathrm{~min}$ at $37^{\circ} \mathrm{C}$. Results are means $\pm \mathrm{SE}, \mathrm{n}=7 . \square$ : sham; $\square:$ CLP. ${ }^{* \star}: \mathrm{p}<0.01$ versus SA uptake; ${ }^{*}: p<0.05$ versus sham.

$(\mathrm{p}<0.01)$ than the association of SA with these cells. Furthermore, the AM isolated from CLP rats had significantly more LA associated with these cells after $60 \mathrm{~min}$ than the macrophages isolated from sham animals.

\section{DISCUSSION}

Although mortality in patients with acute respiratory distress syndrome/acute lung injury has declined over the last decade, it remains high, in the order of 30-60\% [21]. The most common and lethal cause of this disorder is systemic sepsis arising from the gut [22-24]. An important factor contributing to the particularly high mortality associated with sepsis-induced lung injury is the complexity and severity of the disease at the time of treatment. Pulmonary involvement at earlier stages 
of sepsis has not been extensively evaluated, although recent studies using the CLP models of sepsis have shown specific alterations of the endogenous surfactant system when lung dysfunction was relatively mild $[10,15]$. In the present study, the total amount of surfactant and the percentage of LAs was higher in the septic versus sham lungs, mainly due to decreased SAs pool sizes. The total amount of remaining functional forms of LA surfactant was not altered.

To examine the metabolism of surfactant, trace doses of radioactive LAs were instilled into septic rats. The greater radioactivity remaining in LA forms in the CLP animals suggests that there was decreased conversion from LA to SA in the septic rats. In addition, AM from CLP rats were associated with significantly greater amounts of the instilled radiolabel both in vivo and in vitro. It is concluded that both increased uptake of surfactant metabolites via AM and decreased conversion of LA into SA were responsible for the specific alterations of surfactant observed (i.e. decreased SA pools) at this early phase of sepsis-induced lung injury.

The current authors specifically focused on surfactant aggregate metabolism in these animals since these alterations of alveolar surfactant occur relatively quickly after the induction of lung injury. In contrast to the situation in severely injured lungs, the relatively mild lung injury observed in these spontaneously breathing septic animals was associated with no changes in LA pool sizes but a decrease in SA. This resulted in an increased percentage of LA in these spontaneously breathing animals, a finding also reported in a human study evaluating spontaneously breathing children with respiratory infections [12]. The current authors speculate that these changes in the endogenous surfactant system represent a compensatory response of the host to the initial insult. In the current study, more ${ }^{3} \mathrm{H}$-label remained within the airspace in LA forms for the CLP animals compared with sham animals; this is consistent with the concept that decreased conversion of LA into SA is responsible for the altered amounts of surfactant aggregates in the septic lung. One mechanism through which these changes in aggregate conversion may occur is via decreases in the tidal volumes of the CLP animals. At this early stage of lung injury, spontaneously breathing animals have an increased respiratory rate accompanied by an increase in carbon dioxide tension levels. Although the tidal volumes were not measured in these animals, these changes would suggest that the tidal volumes were decreased in the CLP animals. Previous studies have shown that tidal volumes, but not respiratory rates were positively correlated with the conversion of LA into SA [17]. Lower tidal volumes in this setting would, therefore, tend to preserve surfactant in the functional LA forms due to smaller phasic changes in alveolar surface area. Moreover, given the fact that there was relatively little protein leaking into the airspace of these animals, increased protease activity, another major factor driving aggregate conversion, was an unlikely contributor to the aggregate changes observed.

The importance of AM in surfactant metabolism is evident from studies involving transgenic mice deficient in granulocyte macrophage colony stimulating factor $[25,26]$. These animals have marked increases in alveolar surfactant due to the inability of the macrophage to degrade surfactant components.
The current authors showed that within the injured lungs of the CLP rats, AM were associated with greater amounts of the instilled ${ }^{3} \mathrm{H}$-DPPC LA compared with sham groups. In addition, macrophages isolated from the septic rats took up more surfactant lipids in vitro compared with the macrophages from sham animals. Mechanisms responsible for these differences in macrophage activity are unknown, but may be related to increased levels of macrophage inflammatory protein-2 and other inflammatory mediators that are observed in CLP animals.

Although AMs are thought to mainly take up SA forms within the airspace [13, 27], the current in vitro results showed that greater amounts of LAs were associated with the macrophages compared with SAs. However, it should be noted that in vitro conditions, such as the specific localisation of cells with surfactant, as well as the concentrations of surfactant used, may differ from the situation in vivo.

There are some limitations to the present study. These include the fact that tidal volumes in these animals were not measured and it could only be speculated that they were decreased in the CLP group. Measuring tidal volumes in spontaneously breathing animals is technically difficult, and the current authors feel that the assumption is valid given the changes observed in other physiological parameters. Another limitation is that only the macrophages were focused upon in these studies although type II cells and other inflammatory cells recruited to lungs at later stages of injury, such as neutrophils, may also contribute to surfactant changes. Finally, the current authors did not address the role of the surfactant proteins in this study. SP-A has been shown to influence surfactant metabolism, although previous studies, carried out in the authors' laboratories, showed that transgenic mice deficient in SP-A had similar outcomes as wild-types, including surfactant changes, when undergoing the CLP procedure. Evaluation of the other surfactant proteins, particularly their role in treatment strategies for sepsis-induced lung injury, will be the focus of future studies.

In conclusion, the present study suggests that the early changes in surfactant in sepsis-induced lung injury are related to altered surfactant metabolism, including decreased conversion of large aggregates into small aggregates and an increased uptake of surfactant into alveolar macrophages. The authors speculate that these changes may represent a protective response of the host to the primary lung insult by preserving alveolar surfactant in large aggregate forms. However, further studies are required to determine if these changes are indeed beneficial or potentially detrimental in the long term. A greater understanding of the importance of the surfactant changes occurring at the various stages of lung injury, and in particular the earlier stages, is required so that optimal treatment strategies can be developed with the aim of mitigating progressive lung dysfunction.

\section{REFERENCES}

1 Goerke J. Pulmonary surfactant: functions and molecular composition. Biochim Biophys Acta 1998; 1408: 79-89.

2 Lewis JF, Jobe AH. Surfactant and the adult respiratory distress syndrome. Am Rev Respir Dis 1993; 147: 218-233. 
3 Veldhuizen RA, McCaig LA, Akino T, Lewis JF. Pulmonary surfactant subfractions in patients with the acute respiratory distress syndrome. Am J Respir Crit Care Med 1995; 152: 1867-1871.

4 Pison U, Obertacke U, Brand M, et al. Altered pulmonary surfactant in uncomplicated and septicemia-complicated courses of acute respiratory failure. J Trauma 1990; 30: 19-26.

5 Gunther A, Siebert C, Schmidt R. Surfactant alterations in severe pneumonia, acute respiratory distress syndrome, and cardiogenic lung edema. Am J Respir Crit Care Med 1996; 153: 176-184.

6 Gregory TJ, Longmore WJ, Moxley MA. Surfactant chemical composition and biophysical activity in acute respiratory distress syndrome. J Clin Invest 1991; 88: 1976-1981.

7 Gregory TJ, Steinberg KP, Spragg R. Bovine surfactant therapy for patients with acute respiratory distress syndrome. Am J Respir Crit Care Med 1997; 155: 1309-1315.

8 Spragg RG, Gilliard N, Richman P. Acute effects of a single dose of porcine surfactant on patients with the adult respiratory distress syndrome. Chest 1994; 105: 195-202.

9 Anzueto A, Baughman RP, Guntupalli KK. Aerosolized surfactant in adults with sepsis-induced acute respiratory distress syndrome. N Engl J Med 1996; 334: 1417-1421.

10 Malloy J, McCaig L, Veldhuizen R. Alterations of the endogenous surfactant system in septic adult rats. Am J Respir Crit Care Med 1997; 156: 617-623.

11 Putman E, Boere AJF, Van Bree L, Van Golde LMG, Haagsman HP. Pulmonary surfactant subtype metabolism is altered after short-term ozone exposure. Toxicol Appl Pharmacol 1995; 134: 132-138.

12 Mander A, Langton-Hewer S, Bernhard W, Warner JO, Postle AD. Altered phospholipid composition and aggregate structure of lung surfactant is associated with impaired lung function in young children with respiratory infections. Am J Respir Cell Mol Biol 2002; 27: 714-721.

13 Gross NJ. Extracellular metabolism of pulmonary surfactant: the role of a new serine protease. Annu Rev Physiol 1995; 57: 135-150.

14 Lewis JF, Ikegami M, Jobe AH. Altered surfactant function and metabolism in rabbits with acute lung injury. J Appl Physiol 1990; 69: 2303-2310.
15 Malloy J, Veldhuizen RAW, McCormack FX, Korfhagen T, Whitsett J, Lewis J. Pulmonary surfactant and inflammation in septic adult mice: role of surfactant protein A. J Appl Physiol 2002; 92: 809-816.

16 Bailey TC, Cavanagh C, Mehta S, Lewis JF, Veldhuizen RA. Sepsis and hyperoxia effects on the pulmonary surfactant system in wild-type and iNOS knockout mice. Eur Respir J 2002; 20: 177-182.

17 Veldhuizen RAW, Marcou J, Yao L-J, McCaig L, Ito Y, Lewis JF. Alveolar surfactant aggregate conversion in ventilated normal and injured rabbits. Am J Physiol 1996; 270: L152-L158.

18 Gross NJ, Narine KR. Surfactant subtypes of mice: metabolic relationships and conversion in vitro. J Appl Physiol 1989; 67: 414-421.

19 Duck-Chong CG. A rapid sensitive method for determining phospholipid phosphorus involving digestion with magnesium nitrate. Lipids 1979; 14: 492-497.

20 Bligh EG, Dyer WJ. A rapid method of total lipid extraction and purification. Can J Biochem Physiol 1959; 37: 911-917.

21 Abel SJ, Finney SJ, Brett SJ, Keogh BF, Morgan CJ, Evans TW. Reduced mortality in association with the acute respiratory distress syndrome (ards). Thorax 1998; 53: 292-294.

22 Montgomery AB, Stager MA, Carrico CJ, Hudson ED. Causes of mortality in patients with the adult respiratory distress syndrome. Am Rev Respir Dis 1985; 132: 485-489.

23 Angus DC, Linde-Zwirble WT, Lidicker J, Clermont G, Carcillo J, Pinsky MR. Epidemiology of severe sepsis in the United States: analysis of incidence, outcome, and associated costs of care. Crit Care Med 2001; 29: 1303-1310.

24 Livingston DH, Mosenthal AC, Deitch EA. Sepsis and multiple organ dysfunction syndrome: a clinicalmechanistic overview. New Horiz 1995; 3: 257-266.

25 Dranoff G, Crawford AD, Sadelain M. Involvement of granulocyte-macrophage colony-stimulating factor in pulmonary homeostasis. Science 1994; 264: 713-716.

26 Reed JA, Ikegami M, Cianciolo ER. Aerosolized GM-CSF ameliorates pulmonary alveolar proteinosis in GM-CSFdeficient mice. Am J Physiol 1999; 276: L556-L563.

27 Wright JR, Hawgood S. Pulmonary surfactant metabolism. Clin Chest Med 1989; 10: 83-93. 